Draft Version March 5, 2022

Preprint typeset using $\mathrm{LAT}_{\mathrm{E}} \mathrm{X}$ style emulateapj v. 11/10/09

\title{
INITIAL SIZE DISTRIBUTION OF THE GALACTIC GLOBULAR CLUSTER SYSTEM
}

\author{
Jihye Shin ${ }^{1}$, Sungsoo S. Kim ${ }^{1,2}$, SuK-Jin Yoon ${ }^{3}$, And Juhan Kim ${ }^{4}$ \\ Draft version March 5, 2022
}

\begin{abstract}
Despite the importance of their size evolution in understanding the dynamical evolution of globular clusters (GCs) of the Milky Way, studies are rare that focus specifically on this issue. Based on the advanced, realistic Fokker-Planck (FP) approach, we predict theoretically the initial size distribution (SD) of the Galactic GCs along with their initial mass function and radial distribution. Over one thousand FP calculations in a wide parameter space have pinpointed the best-fit initial conditions for the SD, mass function, and radial distribution. Our best-fit model shows that the initial SD of the Galactic GCs is of larger dispersion than today's SD, and that typical projected half-light radius of the initial GCs is $\sim 4.6 \mathrm{pc}$, which is 1.8 times larger than that of the present-day GCs $(\sim 2.5 \mathrm{pc})$. Their large size signifies greater susceptibility to the Galactic tides: the total mass of destroyed GCs reaches $3-5 \times 10^{8} M_{\odot}$, several times larger than the previous estimates. Our result challenges a recent view that the Milky Way GCs were born compact on the sub-pc scale, and rather implies that (1) the initial GCs are generally larger than the typical size of the present-day GCs, (2) the initially large GCs mostly shrink and/or disrupt as a result of the galactic tides, and (3) the initially small GCs expand by two-body relaxation, and later shrink by the galactic tides.

Subject headings: Galaxy: evolution - Galaxy: formation - Galaxy: kinematics and dynamics - globular clusters: general - methods: numerical
\end{abstract}

\section{INTRODUCTION}

Whereas the present-day mass functions (MFs) of globular cluster (GC) systems, which are nearly universal among galaxies (Brodie \& Strader 2006; Jornán et al. 2007), are approximately log-normal with a peak mass $M_{p} \approx 2 \times 10^{5} M_{\odot}$, the MFs of the young massive star cluster (YMC) systems follow a simple power-law distribution (Whitmore \& Schweizer 1995; Zhang \& Fall 1999; de Grijs et al. 2003, among others). Motivated by such a difference between GCs and YMCs, numerous studies have examined the dynamical evolution of the GC MFs to determine whether the initial MFs of GC systems resemble those of YMC systems (Gnedin \& Ostriker 1997; Baumgardt 1998; Vesperini 1998; Fall \& Zhang 2001; Parmentier \& Gilmore 2007; Shin, Kim. \& Takahashi 2008, among others). In particular, Shin, Kim, \& Takahashi (2008, Paper I hereafter) surveyed a wide range of parameter space for the initial conditions of the Milky Way GCs, and considered virtually all internal/external processes: two-body relaxation, stellar evolution, binary heating, galactic tidal field, eccentric orbits and disc/bulge shocks. They found that the initial GC MF that best fits the observed GC MF of the Milky Way is a log-normal function with a peak at $4 \times 10^{5} M_{\odot}$ and a dispersion of 0.33 , which is quite different from the typical MFs of YMCs.

Using the outcome of $N$-body calculations,

jhshin@ap4.khu.ac.kr

${ }^{1}$ Department of Astronomy \& Space Science, Kyung Hee University, Yongin, Kyungki 446-701, Republic of Korea

${ }^{2}$ School of Space Research, Kyung Hee University, Yongin, Kyungki 446-701, Republic of Korea

${ }^{3}$ Department of Astronomy and Center for Galaxy Evolution Research, Yonsei University, Seoul 120-749, Republic of Korea

${ }^{4}$ Center for Advanced Computation, Korea Institute for Advanced Study, 87 Hoegiro Dondaemun-gu, Seoul 130-722, Republic of Korea
Gieles \& Baumgardt (2008) found that the aspect of mass loss in GCs varies with the tidal filling ratio $\Re \equiv r_{h} / r_{J}$, where $r_{h}$ is the half-mass radius and $r_{J}$ is the Jacobi radius. More specifically, the mass loss of GCs in the "isolated regime" $(\Re<0.05)$ is driven mostly by the two-body relaxation, which induces the formation of binaries in the core and causes GCs to expand. On the other hand, the mass loss of GCs in the "tidal regime" ( $\curvearrowright>0.05)$ is influenced by the galactic tides as well, which enables stars in the outer envelope to easily escape (evaporation). Thus, the cluster size $\left(r_{h}\right)$ is as important as the cluster mass $(M)$ and the galactocentric radius $\left(R_{G}\right)$ in determining the dynamical evolution of GCs.

Can YMCs tell us something about the typical initial size of the Milky Way GC system? Observations show that the projected half-light radius $R_{h}$ of YMCs (ages up to $100 \mathrm{Myr}$ ) in the local group ranges between $\sim 2$ and $\sim 30$ pc with a mean value of $\sim 8 \mathrm{pc}$ (Portegies Zwart, McMillan, \& Gieles 2010), which is a few times larger than that of the present-day Milky Way GCs, $R_{h} \sim 2.5$ pc. However, GCs could have formed in different environments and/or by different mechanisms from the YMCs.

Perhaps the best way to estimate the typical size of the GCs is to trace them back to their initial state by calculating their dynamical evolution. In this paper, we study the dynamical evolution of the Galactic GCs and identify the most probable initial conditions not only for the MF and radial distribution (RD), but also the size distribution (SD). Using the same numerical method and procedure as in Paper I, we perform Fokker-Planck (FP) calculations for 1152 different initial conditions (mass, half-mass radius, galactocentric radius and orbit eccentricity), and then search a wide-parameter space for the most probable initial distribution models that evolve into 


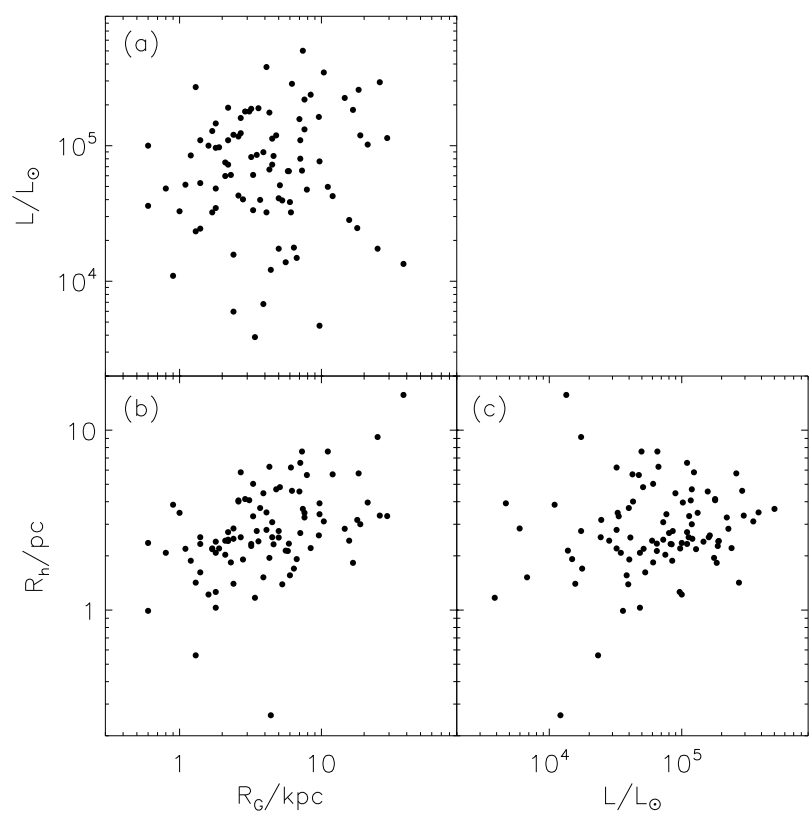

FIG. 1.- Distribution of the Galactic "native" (see the text for definition) globular clusters in the $L-R_{G}$ space (a), $R_{h}-R_{G}$ space (b), and $R_{h}-L$ (c). Data are from the compilation by Harris (1996).

the present-day Galactic GC distributions.

The paper is organized as follows. Section 2 describes the properties of the observed GCs, again which we compare our model results. Section 3 presents models and initial conditions for FP calculations, and Section 4 analyzes the aspects of the size evolution of GCs. We synthesize our FP results in Section 5 to construct the GC system, and examine common features of the best-fit MF, $\mathrm{RD}$, and SD models in Section 6. We discuss characteristics of the final best-fit SD models of the Galactic GCs in Section 7. Finally, conclusions are presented in Section 8.

\section{PRESENT-DAY GC PROPERTIES}

When comparing FP calculations to the present-day Galactic GCs, we consider the "native" GCs only, i.e., "old" halo and bulge/disc clusters, which are believed to be created when a protogalaxy collapses while "young" halo clusters are thought to be formed in external satellite galaxies (Zinn 1993; Parmentier et al. 2000; Mackey \& van den Bergh 2005). Our native GC candidates do not include six objects that belong to the Sagittarius dwarf, seven objects whose origins remain unknown, two objects that have no size information, and fifteen objects that are thought to be the remnants of dwarf galaxies (Lee, Gim, \& Casetti-Dinescu 2007). The total number of our present-day Galactic native GCs is 93 , and their observed properties, such as luminosity $L$, $R_{h}$, and $R_{G}$, were obtained from the database compiled by Harris (1996).

Figure 11 shows scatter plots between observed $L, R_{h}$, and $R_{G}$ values for the 93 Galactic native GCs. The $L$, $R_{h}$, and $R_{G}$ values range between $3.9 \times 10^{3}-5.0 \times 10^{5} L_{\odot}$, $0.3-16 \mathrm{pc}$, and $0.6-38 \mathrm{kpc}$, where the mean values are located at $7.2 \times 10^{4} L_{\odot}, 2.5 \mathrm{pc}$, and $4.1 \mathrm{kpc}$, respectively. The correlation between $R_{h}$ and $R_{G}$ is tighter than the other two correlations (see Figure $1 b$ ). This tight $R_{h}$ $R_{G}$ correlation could be just a result of the initially tight

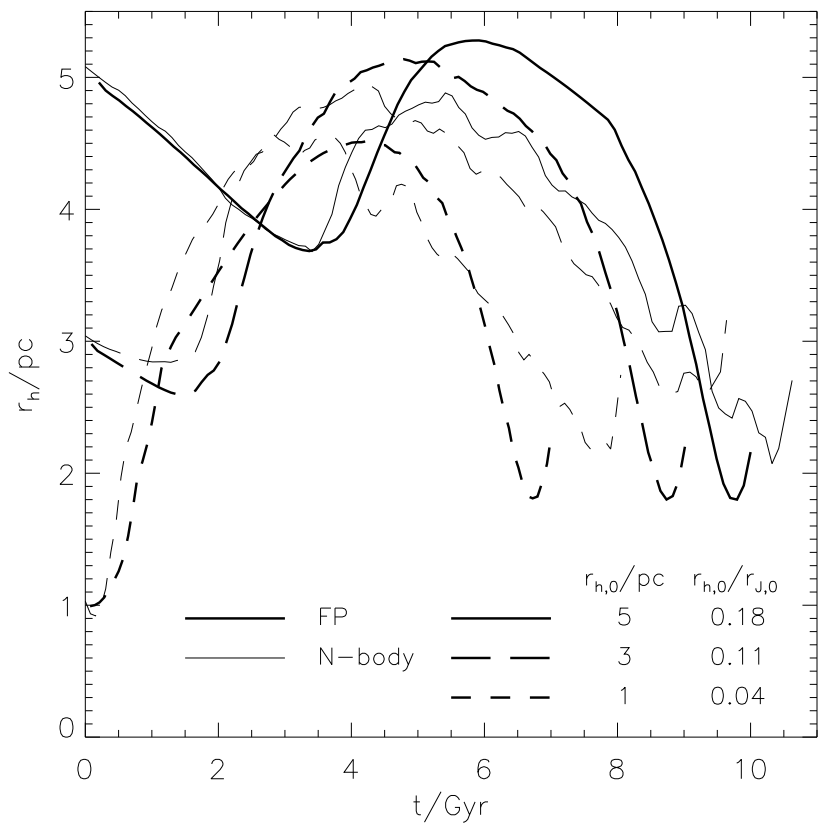

FIG. 2.- Comparison of $r_{h}$ evolution between $N$-body simulations and our FP calculations for GCs with initial conditions of $M=10^{4} M_{\odot}, R_{G}=8.5 \mathrm{kpc}$, and $r_{h}=1,3$, and $5 \mathrm{pc}$ on circular orbits. $N$-body simulations were performed using Nbody 4 code (Aarseth 2003), and mass loss by stellar evolution was not considered in these test calculations (both $N$-body and FP). $r_{h}$ values of the two models agree well within $\sim 20 \%$ during the entire cluster lifetimes.

correlation between $R_{h}$ and $R_{G}$, or it could be due to the preferred disruption of large GCs near the Galactic center (Vesperini \& Heggie 1997; Baumgardt \& Makino 2003). Another possible cause is the expansion of initially small GCs up to $r_{J}$, which is roughly proportional to $R_{G}^{2 / 3}$ for a given GC mass. One of the goals of this paper is to determine which of these possibilities is more feasible.

Previous studies on the evolution of the GC system assumed a certain constant mass-to-light $(M / L)$ ratio, and converted the observed $L$ to $M$ when comparing their numerical values with observations. But the conversion of GC luminosity function (LF) to GC MF using a constant $M / L$ ratio may lead to $M F s$ in error because low-mass stars, which have higher $M / L$ ratios than the high-mass stars, preferentially evaporate from the cluster and this causes the $M / L$ ratio of the cluster to evolve with time (Kruijssen \& Portegies Zwart 2009). For the same reason, there is not a linear relationship between $R_{h}$ and $r_{h}$ among different GCs. Thus, we transform $M$ to $L$, instead of $L$ to $M$, using the stellar mass-luminosity relation of the Padova model (Marigo et al. 2008) with a metallicity of $[\mathrm{Fe} / \mathrm{H}]=-1.16$, which is the mean value for the Galactic native GCs. Our FP calculations, which will be described later, show that the present-day GCs can have $M / L$ ratios ranging between 1.2 and 2.5 and $r_{h} / R_{h}$ ratios ranging between 1.0 and 2.5.

We use dynamical properties such as $M$ and $r_{h}$ when constructing the initial distributions of the Galactic GC system and when calculating the dynamical evolution, while observed quantities, $L$ and $R_{h}$, are used when comparing our FP results with the observations.

\section{MODELS AND INITIAL CONDITIONS}


We adopt the anisotropic FP model used in Paper I, which was originally developed by Takahashi \& Lee (2000, and references therein). The model integrates the orbit-averaged FP equation of two (energy-angular momentum) dimensions and considers multiple stellar mass components, three-body and tidal-capture binary heating, stellar evolution, tidal fields, disk/bulge shocks, dynamical friction, and realistic (eccentric) cluster orbit (see Kim \& Lee (1999) for the tidal binary heating and Paper I for the detailed implementation of dynamical friction and realistic orbits). The model implements the Alternating Direction Implicit (ADI) method developed by Shin \& Kim (2007) for integrating the twodimensional FP equation with better numerical stability.

Parameters for our FP survey are the following four initial cluster conditions: $M, r_{h}$, apocenter distance of the cluster orbit $R_{a}$, and cluster orbit eccentricity $e$. We choose eight $M$ values from $10^{3.5}$ to $10^{7} M_{\odot}$, six $r_{h}$ values from $10^{-1}$ to $10^{1.5} \mathrm{pc}$, and six $R_{a}$ values from $10^{0}$ to $10^{1.67} \mathrm{kpc}$, all equally spaced on the logarithmic scale. For the eccentricity, we choose $e=0,0.25,0.5$, and 0.75 . We perform FP calculations for all possible combinations of these four parameters, thus the total number of cluster models considered in the present study amounts to 1152 .

For the initial stellar mass function (IMF) within each cluster, we adopt the model developed by Kroupa (2001) with a mass range of $0.08-15 \mathrm{M}_{\odot}$, which is realized by 15 discrete mass components in our FP model. Each mass component follows the stellar evolution recipe described by Schaller et al. (1992). The stellar density and velocity dispersion distributions within each cluster follow the King model (King 1966) with a concentration parameter $W_{0}=7$ and with neither initial velocity anisotropy nor initial mass segregation. We use only one value for $W_{0}$, thus the tidal cut-off radius $r_{t}$ of the King profile is proportional to $r_{h}$, while $r_{J}$ varies depending on $M$ and $R_{G}$. Therefore, the Roche lobe filling ratio $\left(r_{t} / r_{J}\right)$ and $\Re$ of our FP models are functions of $r_{h}, M$, and $R_{G}$.

The aspects of mass and size evolution from our FP model are in a good agreement with those from $N$-body methods. A comparison of mass evolution between our FP calculations and the $N$-body simulations performed by Baumgardt \& Makino (2003) for clusters on eccentric orbits with initial masses larger than $10^{4} M_{\odot}$ shows good agreement of cluster lifetimes within $\sim 25 \%$. For a comparison of size evolution, we run a set of $N$-body simulations using Nbody4 code (Aarseth 2003) with $M=10^{4} M_{\odot}, R_{G}=8.5 \mathrm{kpc}$, and $r_{h}=1,3$, and $5 \mathrm{pc}$ (these correspond to $\Re=0.04,0.11$, and 0.18 ), and find that the $r_{h}$ evolutions of the two models agree well within $\sim 20 \%$ during the entire cluster lifetimes (see Figure 2).

Due to the expulsion of the remnant gas from star formation in the pre-gas-expulsion cluster, some of the low-mass pre-gas expulsion clusters can quickly disrupt, and even the surviving low-mass pre-gasexpulsion clusters will lose a significant fraction of their mass within the first several Myr and rapidly expand Baumgardt \& Kroupa 2007; Parmentier \& Gilmore 2007). Since our FP model does not consider the effect of gas expulsion, our initial GC models are to be regarded as models at several Myr after cluster formation.

\section{SIZE EVOLUTION OF INDIVIDUAL GLOBULAR CLUSTERS}

The three main drivers of GC size evolution are the two-body relaxation, the mass loss by stellar evolution, and the galactic tides. In this section, we discuss the size evolution of individual GCs with a subset of our FP calculations. Figure 3 shows the ratios between $r_{h}$ values at the present time (13 Gyr) and at the beginning from our FP calculations as a function of $r_{h, 0}$ and $M_{0}$ for two different $R_{G, 0}$ values (subscripts 0 denote the initial value, hereafter).

Two-body relaxation causes GC core to collapse and the subsequent formation of dynamical binaries in the core makes the whole cluster expand. For GCs that have undergone core collapse in the early phase of evolution, the size of the post-corecollapse expansion follows a scaling relation $r_{h} \propto$ $M_{0}^{-1 / 3} t^{2 / 3}$ Goodman 1984; Kim. Lee, \& Goodman 1998; Baumgardt, Hut, \& Heggie 2002), and thus for a given initial mass and epoch, $r_{h} / r_{h, 0}$ is simply proportional to $r_{h, 0}^{-1}$. Figure 3 indeed shows that the size of the GCs with the same $M_{0}$ tend to converge to a single value $\left(r_{h} / r_{h, 0} \propto r_{h, 0}^{-1}\right)$, if the GCs have small $t_{r h, 0}$ $\left(\log t_{r h, 0} / \mathrm{yr} \lesssim 9\right)$.

Mass loss by stellar evolution causes GCs to adiabatically expand to maintain virialization, and the GC sizes evolve following $r_{h} / r_{h, 0} \propto M_{0} / M$ when the stellar evolution is the main driver of the GC size evolution (Hills 1980). The combination of Kroupa IMF and the stellar evolution recipe described by Schaller et al. (1992) yields a mass loss of $\sim 40 \%$ within 13 Gyr. Thus, GCs would expand by a factor of $\sim 1.67$ as a result of the stellar evolution, if two-body relaxation or the galactic tides are relatively less important in driving the size evolution. Indeed, clusters with $\log t_{r h, 0} / \mathrm{yr} \gtrsim 9$ and $\Re<0.05$ have $r_{h, 13} / r_{h, 0}$ values between 1 and 2 .

While stellar evolution and two-body relaxation cause clusters to expand, galactic tides make clusters shrink in general. A cluster extending farther than $r_{J}$ (overfilling; $r_{t}>r_{J}$ ) loses stars outside $r_{J}$ within a few dynamical timescales, and this naturally causes the mean size of the cluster to decrease. Since $r_{J} \propto R_{G}\left(M / M_{G}\right)^{1 / 3}$ where $M_{G}$ is an enclosed mass of the Milky Way in a given $R_{G}$, the size decrease caused by the galactic tides takes place mostly while the cluster approaches $R_{p}$. The cluster re-expands somewhat by two-body relaxation while approaching $R_{a}$ (Baumgardt \& Makino 2003), but its size gradually decreases while repeating orbital motions. We find that clusters with $0.4<r_{t} / r_{J}<1$ can also shrink moderately as a result of the galactic tides even if it underfills, and clusters initially with $r_{t} / r_{J}<0.4$ (or $\Re<0.05$; i.e., "isolated" GCs) can gradually move into the "tidal" regime as they lose mass or expand by stellar evolution or two-body relaxation. Figure 3 shows that GCs with larger $\Re_{0}$ are smaller at 13 Gyr for a given $M_{0}$ and $R_{G, 0}$, as expected.

Among various initial GC parameters, $r_{h, 0}$ is the most important parameter in the size evolution caused by twobody relaxation $\left(r_{h} / r_{h, 0} \propto M_{0}^{-1 / 3} r_{h, 0}^{-1}\right)$ and that resulting from galactic tides $\left(\Re_{0} \propto M_{0}^{-1 / 3} R_{G}^{-2 / 3} r_{h, 0}\right.$ for a flat rotation curve). For this reason, initially small GCs gen- 




FIG. 3.- Ratios of $r_{h}$ values at $13 \mathrm{Gyr}$ and at the beginning from some of our 1,152 Fokker-Planck calculations as a function of $r_{h}$ at the beginning for two different $R_{G, 0}$ values $(4.6 \mathrm{kpc}$ for the left panel and $46 \mathrm{kpc}$ for the right panel) and four different initial mass $\left(\log M_{0} / M_{\odot}=4,5,6\right.$, and 7$)$. The approximate initial half-mass relaxation times and the initial tidal filling ratios are marked with different colors and symbol sizes, respectively. The blue line indicates the location of $r_{h} / r_{h, 0}=1.67$, which is the expected expansion ratio mainly by the stellar evolution, and the red lines represents the relation $r_{h} / r_{h, 0} \propto r_{h, 0}^{-1}$, which is the expected result when the evolution is dominated by the two-body relaxation.
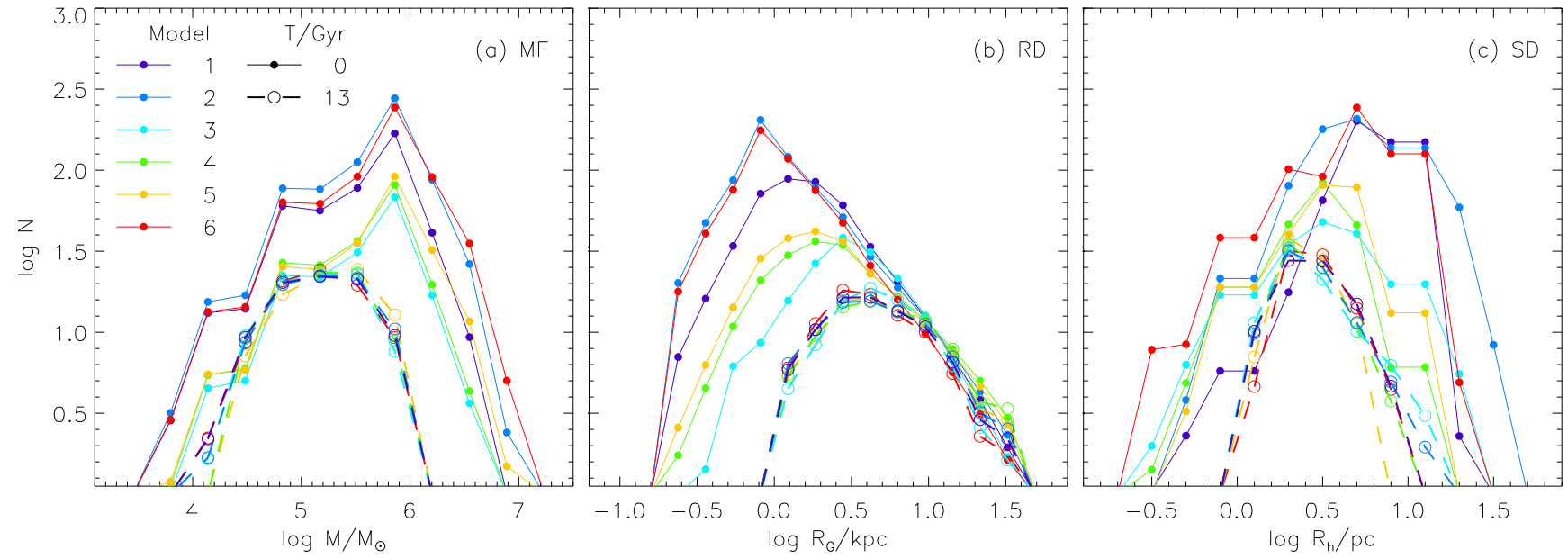

FIG. 4.- Comparison of mass functions (a), radial distributions (b), and size distributions (c) at 13 Gyr (solid lines) and at the beginning (dashed lines) from the best-fit initial parameter set for each SD model.

erally expand (by two-body relaxation), while initially large GCs generally shrink (by the galactic tides) as they evolve. The size evolution of intermediate GCs is determined by more than one dynamical effect, and some GCs can even maintain their initial size over their whole lifetime.

\section{SYNTHESIS OF FOKKER-PLANCK CALCULATIONS}

As discussed in Section 3, we performed a total of 1152 FP calculations with different initial cluster conditions in four-dimensional parameter space, $M, r_{h}, R_{a}$, and $e$. The goal of the present study is to find the initial distribution of these variables that best describe the observed GCs.
For the initial MF model, we adopt a Schechter function,

$$
d N(M) \propto M^{-\alpha} \exp \left(-M / M_{s}\right) d M,
$$

and for the initial RD model, we use a softened power-law function,

$$
d N\left(R_{G}\right) \propto 4 \pi R_{G}^{2} d R_{G} /\left[1+\left(R_{G} / R_{s}\right)^{\beta}\right] .
$$

We assume that the initial MF is independent of initial $R_{G}$. For the sake of simplicity, we do not parameterize the distribution for $e$, and adopt the fixed isotropic distributions, i.e., $d N(e) \propto e d e$. Unlike $M$, the $R_{G}$ of each FP model evolves by oscillating between $R_{p}$ and $R_{a}$, and thus the model RD at 13 Gyr constructed from our population synthesis may suffer from significant random 

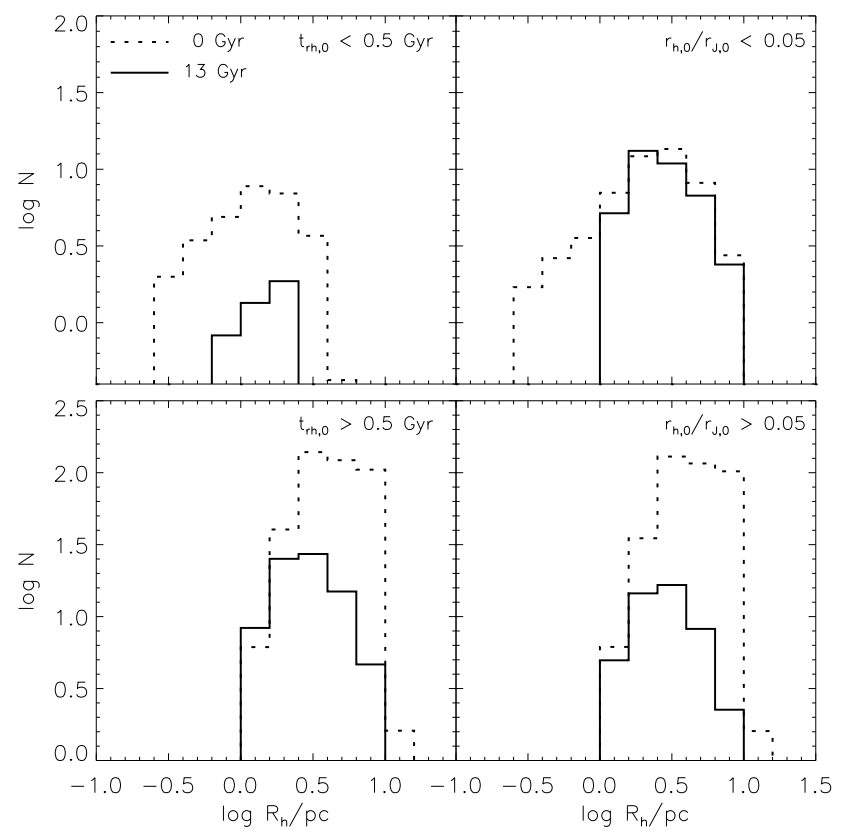

FIG. 5.- Comparison of size distributions at 13 Gyr (solid lines) and at the beginning (dashed lines) from SD Model 1. The upper panels are for initially small GCs $\left(t_{r h, 0}<0.5\right.$ Gyr or $\left.\Re_{0}<0.05\right)$, and the lower panels are for initially large GCs $\left(t_{r h, 0}>0.5\right.$ Gyr or $\left.\Re_{0}>0.05\right)$.

noise. To reduce this noise, we build a model RD by summing the probability distributions between $R_{p}$ and $R_{a}$ that are given by the orbital information at $13 \mathrm{Gyr}$, and we call this a phase-mixed RD. Hereafter, RDs in this paper refer to the phase-mixed RD.

For initial SDs, we use six distribution models (see Table 1). Models 1, 2, and 3 represent a Gaussian distribution of $r_{h}, \rho_{h}$ (mean density within $r_{h}$ ), and $\Re$, respectively, implying that the initial GCs have the preferred initial $r_{h}, \rho_{h}$, and $\Re$, with dispersions. The initial $r_{h}$ of Model 1 does not correlate with the initial $M$ or $R_{G}$, while Models 2 and 3 have initial correlations of $r_{h} \propto M^{1 / 3} \rho_{h}^{-1 / 3}$ and $r_{h} \propto M^{1 / 3} R_{G}{ }^{2 / 3} \Re$. In Models 4,5 , and 6 , the initial $r_{h}$ is determined by powers of initial $M$ and/or $R_{G}$. Note that the power of Model $6\left(r_{h} \propto M^{0.615}\right)$ corresponds to that of the masssize relation derived from the Faber-Jackson relation for early-type galaxies (Faber et al. 1989; Hasegan et. 2005; Gieles et al. 2010).

Once the calculations of the $1152 \mathrm{FP}$ models are done, the aforementioned sets of initial MF, RD, and SD models are used to search for the best-fit parameters in five to seven dimensional space, depending on the SD models (Models 1-6). For this, we synthesize our 1152 FP calculations with appropriate weights to produce a given initial $\mathrm{MF}, \mathrm{RD}$, and $\mathrm{SD}$, and find a set of parameters that best fit the present-day MF, RD, and SD for each of the six SD models. When finding the best set of parameters for each SD model, we minimize the sum of $\chi^{2}$ values from all of the $L, R_{G}$, and $R_{h}$ histograms, which are constructed by using eight bins between $10^{4}$ and $10^{5.8} L_{\odot}$ for $L$, nine bins between $10^{0}$ and $10^{1.6} \mathrm{kpc}$ for $R_{G}$, and nine bins between $10^{-0.6}$ and $10^{1.2} \mathrm{pc}$ for $R_{h}$, all equally spaced on a logarithmic scale. Recall that we use dynamical (theoretical) properties $M$ and $r_{h}$ for set- ting the initial distributions, while observable quantities such as $L$ and $R_{h}$ are used for comparing the models and observations.

\section{BEST-FIT INITIAL DISTRIBUTION OF THE GALACTIC GLOBULAR CLUSTER SYSTEM}

The best-fit parameter sets that minimize the $\chi^{2}$ values between observations and our calculations are presented in Table 2 for the six SD models. We examine the characteristics of our best initial MFs, RDs and SDs in turn.

\subsection{Initial Mass Function}

The best-fit $\alpha$ values for all six SD models are quite low, ranging between 0.01 and 0.07 . The best-fit $\log M_{s} / \mathrm{M}_{\odot}$ values for all six SD models are similar to each other, having values between 5.8 and 5.9. Note that Schechter functions with such small $\alpha$ values are similar to log-normal functions, while those of $\alpha \gtrsim 2$ are closer to power-law functions. Thus, our small $\alpha$ values suggest that log-normal functions better describe the initial MF of the Galactic GC system than power-law functions (see Figure 4 4 ), and this result is consistent with the result of Paper I. One way to explain the log-normallike initial MF is expulsion of the remnant gas due to star formation in the pre-gas-expulsion cluster, which can quickly alter a power-law MF into a log-normal-like MF (Parmentier \& Gilmore 2007). Another possible mechanism resulting in a rapid change in the initial MF is the collisions of clusters with dense clouds or other clusters during the early phase of the galaxy (Elmegreen 2010).

\subsection{Initial Radial Distribution}

Initial RDs from the best-fit parameter sets for all six SD models have similar $\beta$ values $(4.0-4.5)$ but a rather wide range of $R_{s}$ values $(0.3-3.6 \mathrm{kpc})$, and this is consistent with the result of Paper I ( $\beta=4.2$ and $\left.R_{s}=2.9 \mathrm{kpc}\right)$.

Figure $4 b$ shows that most of the GCs that disrupt before $13 \mathrm{Gyr}$ are located in the bulge regime $\left(R_{G, 0}<\right.$ $3 \mathrm{kpc}$ ), and most of the GCs formed in the bulge do not survive until now. We find that only $0.1-8.4 \%$ of the total GC mass initially inside $3 \mathrm{kpc}$ remains in GCs at $13 \mathrm{Gyr}$, and the total stellar mass that escaped from the GCs inside $3 \mathrm{kpc}$ during the last $13 \mathrm{Gyr}$ amounts to $5 \times 10^{7}-3 \times 10^{8} \mathrm{M}_{\odot}$, depending on the SD model.

\subsection{Initial Size Distribution}

The initial SDs from our best-fit parameter sets are of larger dispersion than the present-day SDs for all six SD models (see Figure 4r). The initial SDs evolve into the narrower present-day SDs by two main effects: (1) expansion of GCs with small $r_{h, 0}$, which normally have small $t_{r h, 0}$ and/or small $\Re_{0}$, due to two-body relaxation, and (2) shrinkage (evaporation) of large $r_{h, 0}$ GCs, which normally have large $t_{r h .0}$ and/or large $\Re_{0}$, due to the Galactic tides. Figure [5 shows that the SDs of initially small GCs (upper panels) indeed shift to the larger $r_{h}$ region and those of initially large GCs (lower panels) shift to the smaller $r_{h}$ region after 13 Gyr.

Three $p$-values (significance levels) for $\chi^{2}$ tests of LFs, RDs, and SDs are acceptably high, except for Model 3, which has relatively small $\chi^{2} p$-values for RDs and SDs (see Table 21). However, the high $p$-values from the $\chi^{2}$ 

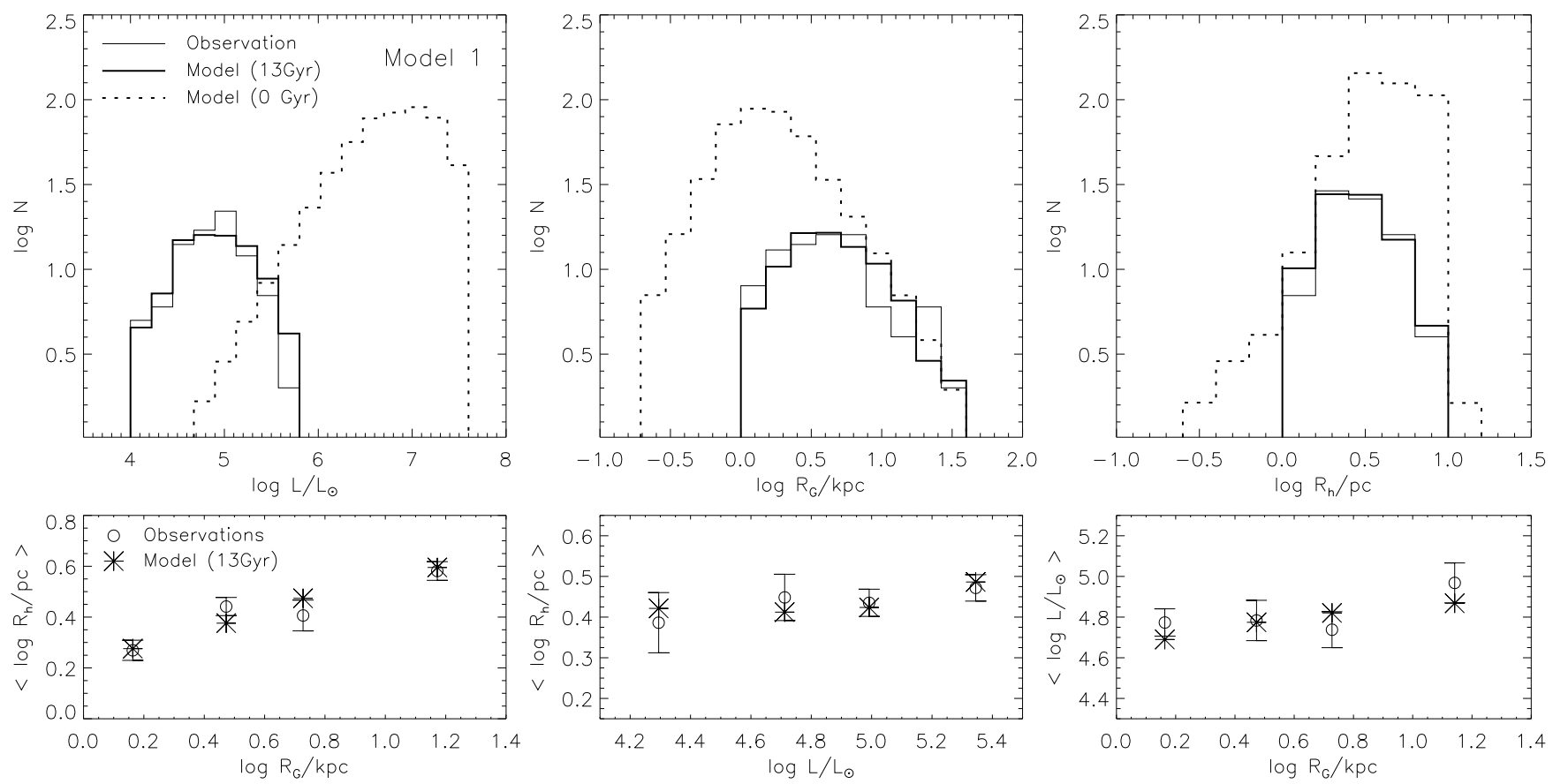

Fig. 6. - L (top left), $R$ (top middle), and $R_{h}$ (top right) histograms at 13 Gyr (thick solid lines) for SD Model 1 with the bestfit parameter set. Also shown together in the upper panels are the corresponding initial distributions (dashed lines) and the observed distributions (thin solid lines). The lower panels show the correlations between $R_{h}$ and $R_{G}$ (bottom left), $R_{h}$ and $L$ (bottom middle), and $L$ and $R_{G}$ (bottom right) relationships for the corresponding best-fit models in the upper panels (asterisks) and from the observations (open circles).


Fig. 7.- Same as Figure 5, but for SD Model 2. 


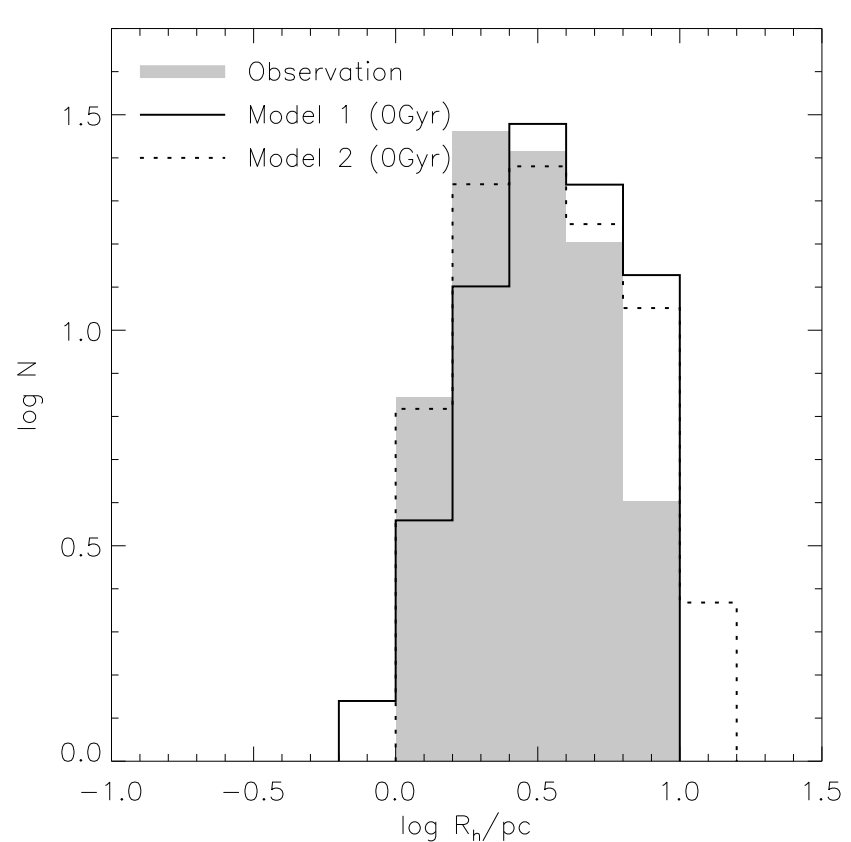

FIG. 8.- Initial SDs of the GCs that survive until 13 Gyr in our best-fit SD models, Models 1 (thick solid line) and 2 (dashed line). Also shown is the currently observed SD (thin solid line with a shaded area). The overall size of the GCs were larger at birth than now even when only the surviving GCs are considered.

tests do not necessarily guarantee that the models with the best-fit parameters restore the observed correlation between $L, R_{G}$, and $R_{h}$ as well. Thus, we implement Student's $t$-tests to see if our models with the best-fit parameters agree with the observed $R_{G}$ dependence of SDs (the $R_{h}-R_{G}$ correlation), the $L$ dependence of SDs (the $R_{h}-L$ correlation), and $R_{G}$ dependence of LFs (the $L-R_{G}$ correlation). For the $R_{h}-R_{G}$ correlation, we calculate $\chi^{2}$ for the difference of $\left\langle\log R_{h}\right\rangle$ and $\sigma_{\log R_{h}}$ between the model and the observation as follows:

$$
\begin{aligned}
\chi^{2}\left(\left\langle\log R_{h}\right\rangle\right) & =\sum_{j} \frac{\left(\left\langle\log R_{h, o, j}\right\rangle-\left\langle\log R_{h, m, j}\right\rangle\right)^{2}}{\sigma_{\log R_{h, m, j}}^{2} / N_{o, j}} \\
\chi^{2}\left(\sigma_{\log R_{h}}\right) & =\sum_{j} \frac{\left(\sigma_{\log R_{h, o, j}}-\sigma_{\log R_{h, m, j}}\right)^{2}}{\sigma_{\log R_{h, m, j}}^{2} / 2 N_{o, j}},
\end{aligned}
$$

where subscripts $o$ and $m$ stand for the observation and the model, respectively, subscript $j$ represents the equal number $R_{G}$ bins, and $\langle\ldots\rangle$ denotes the averaged values. The same calculation is applied to $R_{h}-L$ and $L-R_{G}$ correlation as well. We find that Models 3-6 have $t$-test $p$-values that are too small $(\lesssim 1 \%$ ) for at least one of the $R_{h}-R_{G}, R_{h}-L$, and $L-R_{G}$ correlation. For this reason, we reject Models 3-6 as being a plausible initial SD candidate. Hereafter, we call SD models 1 and 2 "the final best-fit SD models".

Figures 6 and 7 show our two remaining best-fit SD models, a Gaussian distribution of $r_{h}$ (model $1 ; r_{h, c}=$ $6.4 \mathrm{pc}, \sigma_{r_{h}}=2.7 \mathrm{pc}$ ) and a Gaussian distribution of $\rho_{h}\left(\right.$ model $\left.2 ; \rho_{h, c}=690 M_{\odot} p c^{-3}, \sigma_{\rho_{h}}=4.6 M_{\odot} p c^{-3}\right)$. Note that $r_{h, 0}$ values are not correlated with the $R_{G, 0}$ in either model. This implies that the $r_{h, 0}$ of GCs probably does not depend on the strength of the galactic tides. Therefore, we interpret the observed, present-day $R_{h}-R_{G}$ correlation (see Figure 1b) as an outcome of a preferential disruption of the larger GCs at smaller $R_{G}$ due to the Galactic tides.

\section{DISCUSSION}

The typical $R_{h, 0}$ value from our final best-fit SD models (Models 1 and 2) is $\sim 4.6 \mathrm{pc}\left(r_{h, 0} \sim 7 \mathrm{pc}\right.$ ), and this is 1.8 times larger than that of the present-day GCs $(\sim 2.5 \mathrm{pc})$. This result is rather different from a recent argument by Baumgardt et al. (2010) that most GCs were born compact with $r_{h, 0}<1 \mathrm{pc}$. Our result implies that GCs initially have a rather wide SD, the typical value of which is similar to that of YMCs in parsec scale, and have evolved to have a narrower SD with a smaller mean value.

We also find that GCs formation favors a "tidal" environment over an "isolated" environment. The number of tidal GCs $\left(\Re_{0}>0.05\right)$ at 0 Gyr from our final best-fit SD models is approximately five times larger than that of isolated GCs $\left(\Re_{0}<0.05\right)$. The ratio of tidal to isolated GCs, however, drastically decreases as GCs evolve because tidal GCs are more easily disrupted, and this ratio becomes $\sim 0.2$ at 13 Gyr.

Figure 8 shows the initial SDs of the GCs that survive until 13 Gyr in Models 1 and 2. We find that these initial SDs are broader $\left(\sigma\left(R_{h}\right)=2.1\right.$ and $2.5 \mathrm{pc}$, respectively) and centered at higher values $\left(\overline{R_{h}}=4.1\right.$ and $\left.4.0 \mathrm{pc}\right)$ than the currently observed $\mathrm{SD}\left(\sigma\left(R_{h}\right)=1.2 \mathrm{pc}, \overline{R_{h}}=\right.$ $2.5 \mathrm{pc})$. Thus, the overall size of the GCs were larger at birth than now by a factor of $\sim 2$ even when only the surviving GCs are considered.

The initial total masses in $\operatorname{GCs}\left(M_{t, 0}\right)$ of the final best-fit SD models are $2.8 \times 10^{8} M_{\odot}$ (Model 1) and $5.3 \times 10^{8} M_{\odot}$ (Model 2), and the masses that have left the GCs during the lifetime of the Galaxy $\left(\Delta M_{t}\right)$ are $2.5 \times 10^{8} M_{\odot}\left(\right.$ Model 1) and $5.0 \times 10^{8} M_{\odot}$ (Model 2). These give $\Delta M_{t} / M_{t, 0}$ values of 0.89 and 0.94 for Models 1 and 2 , respectively. Our $\Delta M_{t}$ values are several times larger than previous estimates made by Baumgardt (1998, 4.0-9.5 $\left.\times 10^{7} M_{\odot}\right)$, Vesperini $\left(1998,5.5 \times 10^{7} M_{\odot}\right)$, and Paper I $\left(1.5-1.8 \times 10^{8} M_{\odot}\right)$. Our larger $\Delta M_{t}$ values are due to the facts that (1) we consider virtually all disruption mechanisms in the calculations for the dynamical evolution of individual GCs, and (2) we use more a flexible initial $r_{h}$ distribution, which can have a relatively larger fraction of GCs with a large $r_{h}$ (larger GCs are more vulnerable to the galactic tide). Note that $\Delta M_{t}$ will be larger if one considers the clusters that have been disrupted in the process of remnant gas expulsion.

We note that contrary to the finding in the present paper, detailed dynamical modeling of individual clusters shows that at least some of the clusters must have started with a very small size. For example, Monte Carlo calculations by Heggie \& Giersz (2008) and Giersz \& Heggie (2009, 2011) find $0.58 \mathrm{pc}, 0.40 \mathrm{pc}$, and $1.9 \mathrm{pc}$ as best-fit initial $r_{h}$ values for the observed current states of M4, NGC 6397, and 47 Tuc, respectively. These values are several times smaller than the typical initial $r_{h}$ found for the Galactic GC system from our calculations, $\sim 7 \mathrm{pc}$. However, we also note that the Monte Carlo models used for these three clusters all assume circular cluster orbits while M4 and NGC 6397 have moderate to high orbit eccentricities (0.82 and 0.34 , respectively). We have performed several FP calculations for these two clusters and 
find that consideration of appropriate eccentric orbits can increase the best-fit initial $r_{h}$ by a factor of $3-5$.

\section{SUMMARY}

We have calculated the dynamical evolution of Galactic GCs using the most advanced and realistic FP model, and searched a wide parameter space for the best-fitting initial SD, MF, and RD models that evolve into the present-day distribution. We found the initial MF of the Galactic GC system is similar to the log-normal function rather than the power-law function, and the RD of the GC system undergoes significant evolution inside $R_{G}=3 \mathrm{kpc}$ through the strong Galactic tides. We also found that the initial SD of the GC system evolves to narrower present-day SDs through two effects: shrinkage of large GCs by the galactic tides and expansion of small GCs by two-body relaxation. The typical initial projected half-mass radius from the final best-fit model, $\sim 4.6 \mathrm{pc}$, is 1.8 times larger than that of the present-day value, $\sim 2.5 \mathrm{pc}$. The ratio of "tidal" GCs to "isolated" GCs is $\sim 5$ at 0 Gyr and decreases down to $\sim 0.2$ at 13 Gyr.

Since tidal GCs are found to be dominant in the beginning, one might expect the initial size of the GCs to be correlated with the Jacobi radius, i.e., to be a function of the galactocentric radius. However, our final best-fit SD models (Models 1 and 2) do not seem connected to the galactocentric radius. This implies that the GC formation process favors a certain size and density, regardless of the tidal environment. Such a $R_{G}$-independent initial $\mathrm{SD}$ evolves into a present-day SD, which shows a tight $r_{h}-R_{G}$ correlation through evaporation and two-body relaxation.

We thank Holger Baumgardt and Mark Gieles for helpful discussion. This work was supported by Basic Science Research Program (No. 2011-0027247) through the National Research Foundation (NRF) grant funded by the Ministry of Education, Science and Technology (MEST) of Korea. This work was partially supported by WCU program through NRF funded by MEST of Korea (No. R31-10016). J.S. deeply appreciates Koji Takahashi for the help with his FP models. S.J.Y. acknowledges support by the NRF of Korea to the Center for Galaxy Evolution Research and by the Korea Astronomy and Space Science Institute Research Fund 2011 and 2012. S.J.Y. thanks Daniel Fabricant, Charles Alcock, Jay Strader, Nelson Caldwell, Dong-Woo Kim, and Jae-Sub Hong for their hospitality during his stay at Harvard-Smithsonian Center for Astrophysics as a Visiting Professor in 20112012 .

\section{REFERENCES}

Aarseth, S. J. 2003, Gravitational N-body Simulations, Cambridge University Press

Baumgardt, H. 1998, A\&A, 330, 480

Baumgardt, H., Hut, P., \& Heggie, D. C. 2002, MNRAS, 336, 1069

Baumgardt, H., \& Makino, J. 2003, MNRAS, 340, 227

Baumgardt, H., \& Kroupa, P. 2007, MNRAS, 380, 1589

Baumgardt, H., Parmentier, G., Gieles, M., \& Vesperini, E. 2010, MNRAS, 401,1832

Brodie, J. P., \& Strader, J. 2006, ARA\&A, 44, 193

de Grijs, R., Fritze-v. Alvensleben, U., Anders, P., Gallagher, J. S., Bastian, N., Taylor, V. A., \& Windhorst, R. A. 2003, MNRAS, 342, 259

Elmegreen, B. G. 2010, ApJ, 712, L184

Faber, S. M., Wegner, G., Burstein, D., Davies, R. L., Dressler, A., Lynden-Bell, D., \& Terlevich, R. J. 1989, ApJS, 69, 763

Fall, M., \& Zhang, Q. 2001, ApJ, 561, 751

Gieles, M., \& Baumgardt, H. 2008, MNRAS, 389, L28

Gieles, M., Baumgardt, H., Heggie, D. C., \& Lamers, H. J. G. L. M. 2010, MNRAS, 408, 16

Giersz, M., \& Heggie, D. C. 2009, MNRAS, 395, 1183

Giersz, M., \& Heggie, D. C. 2011, MNRAS, 410, 2698

Gnedin, O. Y., \& Ostriker, J. P. 1997, ApJ, 474, 223

Goodman, J. 1984, ApJ, 280, 298

Harris, W. E. 1996, AJ, 112, 1487

Haşegan, M., Jordán, A., Côté, P., Djorgovski, S. G., McLaughlin, D. E., Blakeslee, J. P., Mei, S., West, M. J., Peng, E. W., Ferrarese, L., Milosavljević, M., Tonry, J. L., \& Merritt, D. 2006, ApJ, 627, 203

Heggie, D. C., \& Giersz, M. 2008, MNRAS, 389, 1858

Hills, J. G. 1980, ApJ, 235, 986
Jordán, A., McLaughlin,D. E., Côté, P., Ferrarese, L., Peng, E. W., Mei, S., Villegas, D., Merritt, D., Tonry, J. L., \& West, M. J. 2007, ApJS, 171, 101

Kim, S. S., Lee, H. M., \& Goodman, J. 1998, ApJ, 495, 786

Kim, S. S., \& Lee, H. M. 1999, A\&A, 347, 123

King, I. R. 1966, AJ, 71, 64

Kroupa, P. 2001, MNRAS, 322, 231

Kruijssen, J. M. D., \& Portegies Zwart, S. F. 2009, AJ, 698, L158

Lee, Y.-W., Gim, H. B., \& Casetti-Dinescu, D. I. 2007, ApJ, 661, 49

Mackey, A. D., \& van den Bergh, S. 2005, MNRAS, 360, 631

Marigo, P., Girardi, L., Bressan, A., Groenewegen, M. A. T., Silva, L., \& Granato, G. L. 2008, A\&A, 482, 883

Parmentier, G., \& Gilmore, G. 2007, MNRAS, 377, 352

Parmentier, G., Jehin E., Magain P., Noels, A., \& Thoul, A. A. 2000, A\&A, 363, 526

Portegies Zwart, S. F., McMillan, S. L. W., \& Gieles, M. 2010, ARA\&A, 48, 431

Schaller, G., Schaerer, D., Meynet, G., \& Maeder, A. 1992, A\&A, 96, 269

Shin, J., \& Kim, S. S. 2007, JKAS, 40, 91

Shin, J., Kim, S. S., \& Takahashi, K. 2008, MNRAS, 386, L67 (Paper I)

Takahashi, K., \& Lee, H. M., 2000, MNRAS, 316, 671

Tremaine, S. D., Ostriker, J. P., \& Spitzer, L. Jr. 1975, ApJ, 196, 407

Vesperini, E., \& Heggie, D. C. 1997, MNRAS, 289,898

Vesperini, E. 1998, MNRAS, 299, 1019

Whitmore, B.C., \& Schweizer, F., 1995, ApJ, 109, 960

Zhang, Q., \& Fall, M. 1999, ApJ, 527, 81

Zinn, R. 1993, ASPC, 48, 39 
TABLE 1

INITIAL SD MODELS

\begin{tabular}{cll}
\hline SD model & Functional form & Parameters \\
\hline 1 & $\mathcal{N}\left(r_{h, c}, \sigma_{r h}^{2}\right)^{\mathrm{a}}$ & $\alpha, M_{s}, \beta, R_{s}, r_{h, c}, \sigma_{r_{h}}$ \\
2 & $\mathcal{N}\left(\rho_{h, c}, \sigma_{\rho_{h}}^{2}\right)^{\mathrm{a}}$ & $\alpha, M_{s}, \beta, R_{s}, \rho_{h, c}, \sigma_{\rho_{h}}$ \\
3 & $\mathcal{N}\left(\Re_{c}, \sigma_{\Re}^{2}\right)^{\mathrm{a}}$ & $\alpha, M_{s}, \beta, R_{s}, \Re_{c}, \sigma_{\Re}$ \\
4 & $r_{h}=\kappa M^{\lambda} R^{\nu}$ & $\alpha, M_{s}, \beta, R_{s}, \kappa, \lambda, \nu$ \\
5 & $r_{h}=\kappa M^{\lambda}$ & $\alpha, M_{s}, \beta, R_{s}, \kappa, \lambda$ \\
6 & $r_{h}=\kappa M^{0.615}$ & $\alpha, M_{s}, \beta, R_{s}, \kappa$ \\
\hline
\end{tabular}

TABLE 2

BEST-FIt PARAMETERS FOR INITIAL GC Distributions

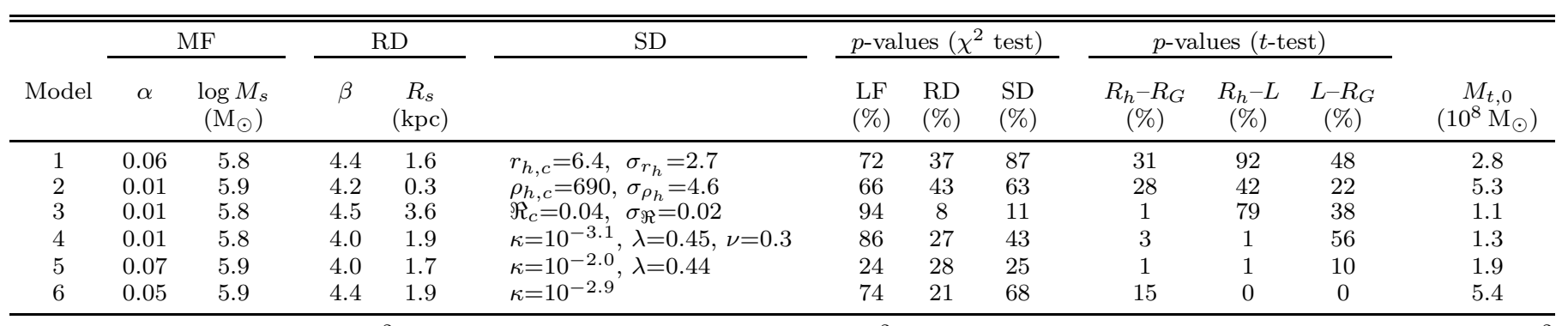

NoтE. - The $p$ value for the $\chi^{2}$ test ( $t$-test) is the probability of having a $\chi^{2}(t)$ value that is larger than the value obtained from our $\chi^{2}$ $(t)$ test between the model and the observation, whose degree of freedom is 8 or $9(4) . r_{h}$ and $r_{h, c}$ are in units of pc, and $\rho_{h}$ and $\rho_{h, c}$ are in units of $\mathrm{M}_{\odot} / \mathrm{pc}^{3}$. 